\title{
Quantum signatures in the stabilization dynamics
}

\author{
J. B. Watson, ${ }^{1}$ C. H. Keitel, ${ }^{2}$ P. L. Knight, ${ }^{2}$ and K. Burnett ${ }^{1}$ \\ ${ }^{1}$ Clarendon Laboratory, Department of Physics, University of Oxford, Parks Road, Oxford OX1 3PU, United Kingdom \\ ${ }^{2}$ Blackett Laboratory, Optics Section, Imperial College, Prince Consort Road, London SW7 2BZ, United Kingdom
}

(Received 26 May 1995)

\begin{abstract}
We investigate the phase-space dynamics of a hydrogenic atom in a very intense, high-frequency laser field, by comparing the time-dependent quantum Wigner functions with the corresponding classical distributions calculated using the Monte Carlo method. In both cases we model the atom by a one-dimensional soft-core potential in order to simplify the calculation. We demonstrate the importance of nonclassical interferences in the Wigner function and show how negative parts of it observed at the peak of the pulse are associated with the formation of a coherent superposition of dressed "Kramers-Henneberger" $(\mathrm{KH})$ eigenstates. These signatures of the quantum dynamics are eliminated towards the end of the pulse when the coherence between the KH eigenstates degrades so that the classical and the quantum mechanical phase-space distributions come into agreement.

PACS number(s): $32.80 \mathrm{Rm}, 42.50 \mathrm{~Hz}, 42.65 \mathrm{Ky}$
\end{abstract}

The availability of intense femtosecond lasers has led to the discovery of a whole range of nonperturbative phenomena in the interaction of atoms with laser fields. These phenomena include multiphoton ionization, above threshold ionization, harmonic generation, and more recently stabilization. By stabilization we mean the reduction of the ionization rate of an atom with increasing laser intensity for intense, highfrequency, laser fields. This effect has been predicted by many quantum-mechanical models [1-4], and has recently been observed experimentally [5]. These intense field effects have been extensively studied, both experimentally [6] and theoretically, and have been the subject of several review articles [7]. To date most of the calculations done in this intense field regime have been numerical, using either the time-dependent Schrödinger equation (first in one dimension, and later in three dimensions), or time-independent methods such as Floquet theory [8]. While both of these approaches give good predictions of the single-atom response, they give little insight into the underlying physics of the process. In addition, both methods are very computer intensive, and therefore hard to include in fully self-consistent models of the macroscopic response [9]. These limitations have motivated the search for alternative models of the dynamics of atoms in intense fields, in particular classical or semiclassical models of the atomic response [10]. In the low-frequency regime a semiclassical model consisting of a tunneling process followed by a quiver-induced "recollision" has been very successful in interpreting the plateau and cutoff of the harmonic spectra as well as scaling laws for harmonic generation efficiency [11]. The relevance of classical dynamics in the low-frequency limit has, therefore, been clearly established. There has also been a fair amount of discussion of classical models in the high-frequency stabilization regime. The question of whether this effect is classical or quantum mechanical in nature has previously been addressed by Bestle, Akulin, and Schleich [12] using a phase-space description of the dynamics. They predicted that for a sudden turn-on of the field the process was essentially classical, while for an adiabatic turn-on the process was purely quantum in nature.
In this paper we study the stabilization dynamics of a hydrogen atom in an intense pulsed field using quantum and classical phase-space distributions. In the quantum case we solve the time-dependent Schrödinger equation in one dimension in order to calculate time-dependent wave functions. We then use these wave functions to calculate the Wigner function [13] for the system. In the classical case we take a distribution of points in phase space corresponding to the classical [one-dimensional (1D)] orbit, and then solve the classical equation of motion for each set of initial conditions. We find three distinct regions during the evolution of the pulse. Near the start of the pulse we find that both distributions are dominated by the initial conditions and there is little agreement. At the peak of the pulse we find total disagreement between the quantum and classical models, with the quantum Wigner function exhibiting large negative areas, indicating quantum interference effects. Towards the end of the pulse we find remarkable agreement between the quantum and classical distributions.

In order to calculate the quantum-mechanical phase-space distribution we solve the time-dependent Schrödinger equation in one dimension. For numerical and conceptual reasons it is convenient to perform the calculation in the KramersHenneberger $(\mathrm{KH})$ reference frame, a noninertial frame where the "quiver motion" of the electron is zero [14]. The wave function in the $\mathrm{KH}$ frame $\psi_{\mathrm{KH}}(q, t)$ is related to that in the length gauge by a unitary transformation, and obeys the partial differential equation:

$$
i \hbar \partial_{t} \psi_{\mathrm{KH}}(q, t)=\left[-\frac{\hbar^{2} \partial_{q}^{2}}{2 m}+V(q+\alpha(t))\right] \psi_{\mathrm{KH}}(q, t)
$$

where $V(q)$ is the potential due to the nucleus, and

$$
\alpha(t)=-\frac{e}{m} \int_{0}^{t} d t^{\prime} A\left(t^{\prime}\right)
$$

is the displacement of a (classical) free electron in the field $E(t) . A(t)$ is the vector potential corresponding to our pulsed electric field, which we write as $E(t)$ 
$=E_{0} h(t) \cos (\omega t)$ describing a pulse of peak intensity $E_{0}$ modulated with an envelope function $h(t)$

$$
h(t):=\left\{\begin{array}{l}
\sin ^{2}[\omega t /(4 c)] \text { if } 0<t<2 t_{\max }:=4 \pi c / \omega \\
0 \text { otherwise }
\end{array}\right.
$$

where $c$ and $t_{\max }$ correspond to the number of cycles and the time required to reach the maximal intensity of the pulse.

In this calculation we use the soft-core (or Rochester) potential scaled to have its lowest eigenstate at the same energy as the ground state of hydrogen

$$
V(q)=-\frac{e^{2}}{\sqrt{2 a_{0}^{2}+q^{2}}},
$$

where $a_{0}$ is the radius of the first Bohr orbit.

The use of this 1D potential for calculations in the stabilization regime has previously been discussed by Ménis et al. [15] and by Millack [16]. Their conclusion is that the singularity in the $3 \mathrm{D}$ Coulomb potential increases the ionization rate. Millack also shows that so long as one takes into account the increased ionization in the $3 \mathrm{D}$ case, the agreement between the $1 \mathrm{D}$ and $3 \mathrm{D}$ cases is very good. We therefore believe that the qualitative behavior of the system, and therefore the conclusions we present in this paper, would be unchanged had we used a 3D Coulomb potential.

We obtain the wave function $\psi$ in the length gauge via the inverse Kramers-Henneberger transformation:

$$
\begin{aligned}
\psi(q, t)= & U^{\dagger}(t) \psi_{\mathrm{KH}}(q, t) \\
= & \exp \left[-i \frac{e}{\hbar} q A(t)\right] \exp \left[-i \frac{e}{m \hbar} \int_{0}^{t} d t^{\prime}\left[-A\left(t^{\prime}\right) p\right.\right. \\
& \left.\left.+e A^{2}\left(t^{\prime}\right) / 2\right]\right] \psi_{\mathrm{KH}}(q, t)
\end{aligned}
$$

The wave function gives us complete information about the state of the system at any time, we use it to calculate the corresponding Wigner function [13], given by

$$
\begin{aligned}
W(q, p, t)= & \frac{1}{\pi \hbar} \int_{-\infty}^{+\infty} d q^{\prime} \psi\left(q-q^{\prime}, t\right) \psi^{*}\left(q+q^{\prime}, t\right) \\
& \times \exp \left[2 i q^{\prime} p / \hbar\right] .
\end{aligned}
$$

From this definition we can see that the Wigner function depends on time, and also on the spatial and momentum phase space variables $q$ and $p$.

In the corresponding classical calculation we start from the classical Hamiltonian:

$$
H=\frac{1}{2} m\left(\frac{d q}{d t}\right)^{2}-e E_{0} q h(t) \cos (\omega t)+V(q) .
$$

From this we obtain a Newtonian equation of motion for the laser field and soft-core potential

$$
m \frac{d^{2} q(t)}{d t^{2}}=e\left[E_{0} h(t) \cos (\omega t)-\frac{e q(t)}{\left[2 a_{0}^{2}+q(t)^{2}\right]^{3 / 2}}\right] .
$$

This second-order equation of motion requires two initial conditions. To compare with quantum-mechanical dynamics, which originates from spatially spread wave functions rather than precisely defined points in space and momentum, we have performed an averaging over the initial conditions using a standard Monte Carlo method [17]. In this onedimensional case we evaluate the positions and momenta of the field-free evolving electron along one cycle around the nucleus, at a set of times all separated by a constant amount, and then take these as initial conditions for the motion of the electron in the field due to the nucleus and external laser field. This means using a microcanonical distribution with an equal distribution in phase space; thus for the phase-space distribution as a function of the energy $\mathscr{E}$ :

$$
\rho(\mathscr{E}) \propto \delta\left(\mathscr{E}-\mathscr{E}_{0}\right)
$$

where $\mathscr{E}_{0}=-13.6 \mathrm{eV}$ is the initial energy of the ground-state hydrogenic electron due to its initial position in the binding potential and its kinetic energy.

For every member of this ensemble of initial conditions, we can solve Eq. (9) and thus obtain the spatial and momentum coordinate of the hydrogenic electron at any time during the interaction with the nucleus. This gives rise to a classical phase-space distribution that we may compare with quantum phase-space distributions, of which the Wigner function is the most interesting example. This classical calculation produces a collection of points in phase space, rather than a continuous distribution, as one obtains from the Wigner function. In order to obtain a continuous distribution, one can take the convolution of this collection of points with a Gaussian. If we compare this smoothed classical distribution with quantum phase-space distributions, the amount of smoothing needs to be addressed. A smoothing of $\hbar$ in both phase-space parameters would just take account of Heisenberg's minimal uncertainty condition and would render the classical phase-space distribution more similar to the intrinsically quantum-mechanical result. We cannot of course reproduce the full quantum result; this is clearly demonstrated by the large negative areas in the Wigner function, which we observe and which are not possible in the smoothed classical phase-space distribution. However, when convolved with a Gaussian of appropriate width $(\hbar)$ the Wigner function is smoothed to the Husimi or $Q$ function, which is always positive. Thus, the Husimi function is expected to resemble more closely a classical distribution with a smoothing of $\hbar$. In this paper we are interested in identifying quantum signatures in the phase-space dynamics and therefore choose representations where the differences between the classical and quantum dynamics are most pronounced. We have therefore decided to present the classical results in their bare form as a distribution of points in phase space. In the quantum case we stick to the Wigner function, and do not show any other quasiprobability distribution.

Figure 1 shows the full Wigner function (a) and its contour plot (b) after 6 cycles of our pulse. The most striking feature of this plot is the "fringe structure" observed close to the nucleus. In this paper we use the term fringes to describe the rays observed in the Wigner function. Since the term fringes is most commonly associated with the characteristic patterns produced by interference, we should emphasize that the fringe structures we refer to do not arise from interference. Such structures have previously been observed in clas- 
(a) 6 cycles

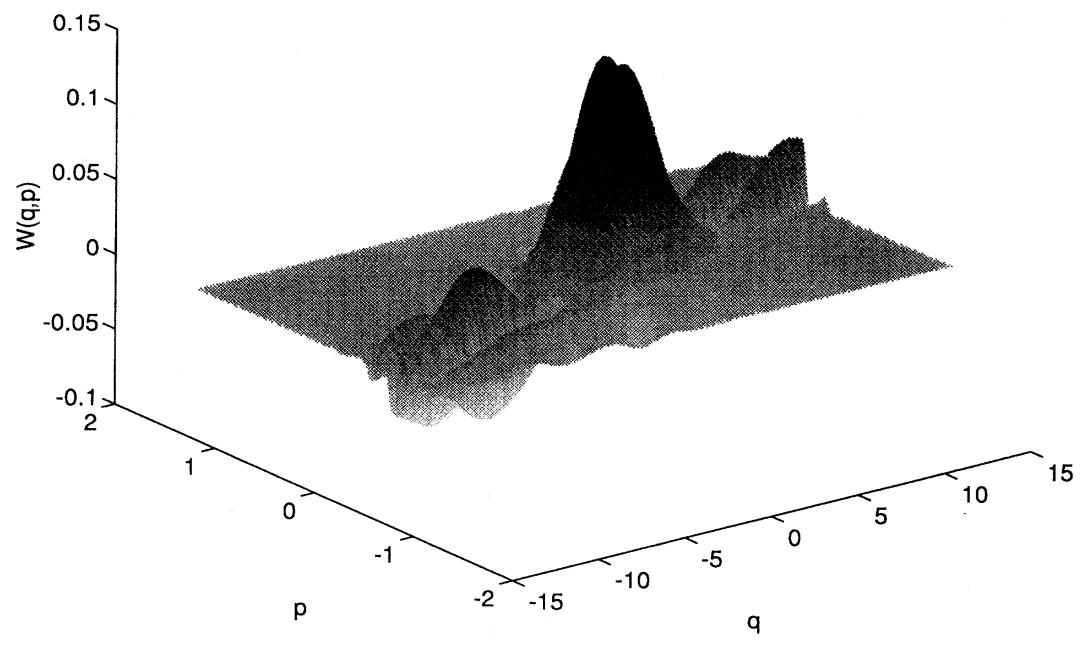

(b) 6 cycles

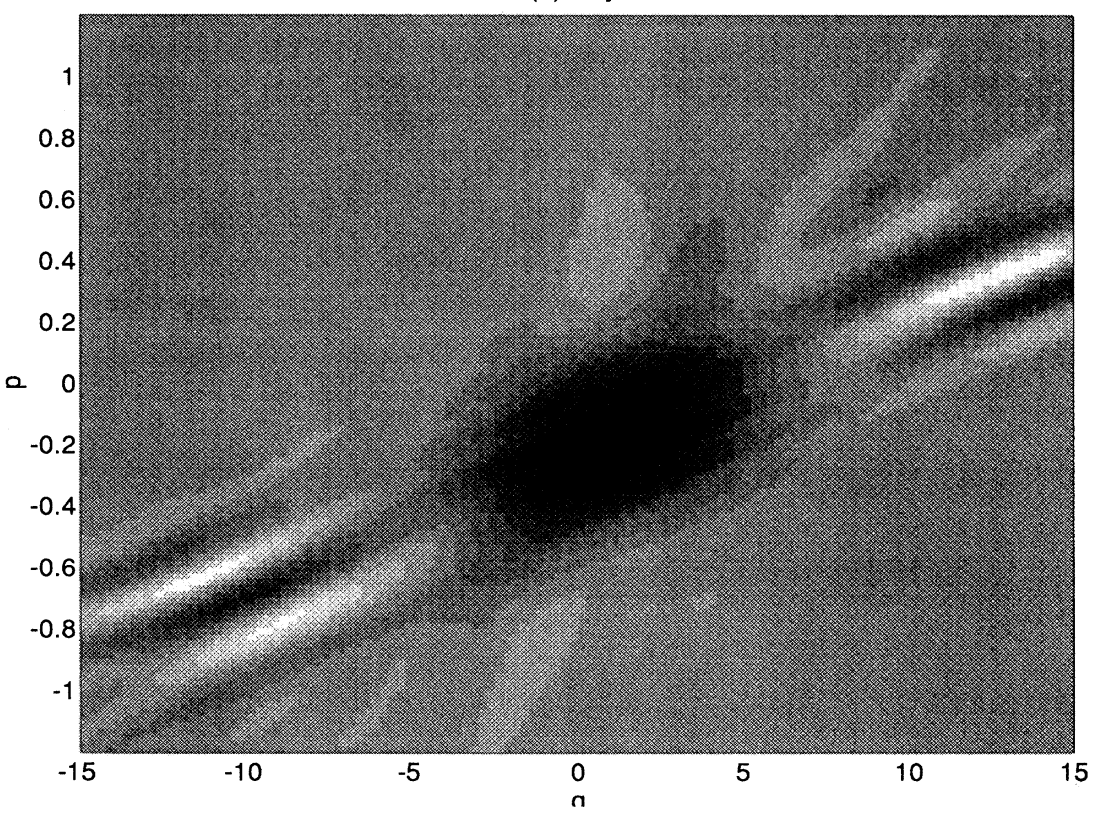

FIG. 1. The $3 d$ Wigner function (a) and its contour (b) of the hydrogenic electron after 6 cycles of the application of a pulsed laser field with 12 turn-on cycles, 12 turn-off cycles, maximal electric field strength $E_{0}=10$ a.u. and, angular frequency $\omega=1$ a.u. The quantummechanical calculations leading to these results are based on the dynamics of the hydrogenic electron in the soft-core potential as described by the corresponding Schrödinger equation. sical simulations of atomic behavior in the stabilization regime by Grobe and Law [4], and are associated with ionization dynamics of the atom. The interpretation of the fringes is that the ionization occurs at discrete times in the evolution of the atom. Each fringe corresponds to emission with a distribution of velocities at a given instant in time. It follows from classical mechanics that the gradient of each fringe corresponds to the inverse of the time elapsed since the emission. Although there is a clear physical description of the fringe structure in terms of classical mechanics, the distribution obtained by means of our classical calculation is still dominated by the initial Monte Carlo distribution of the electron dynamical variables at this early stage of the pulse so that the tunneling and diffusion play essentially no role.
Therefore the agreement between the quantum and classical results is only marginal. We have therefore refrained from presenting the classical results at this time step and shall show that towards the end of the pulse these fringe structures occur in both the quantum and classical distributions.

When we reach the peak of the pulse, we find complete disagreement between the classical and quantum models. This is shown in Fig. 2 where we plot the quantum (a) and classical (b) distributions after 12 cycles of our 24 cycle pulse. In the quantum case the fringe structure that we observed in Fig. 1 is at a greater distance from the nucleus. From this we conclude that the ionization rate was more rapid earlier in the pulse, in other words we have observed stabilization of the atom. In the quantum case there are two 
(a) 12 cycles

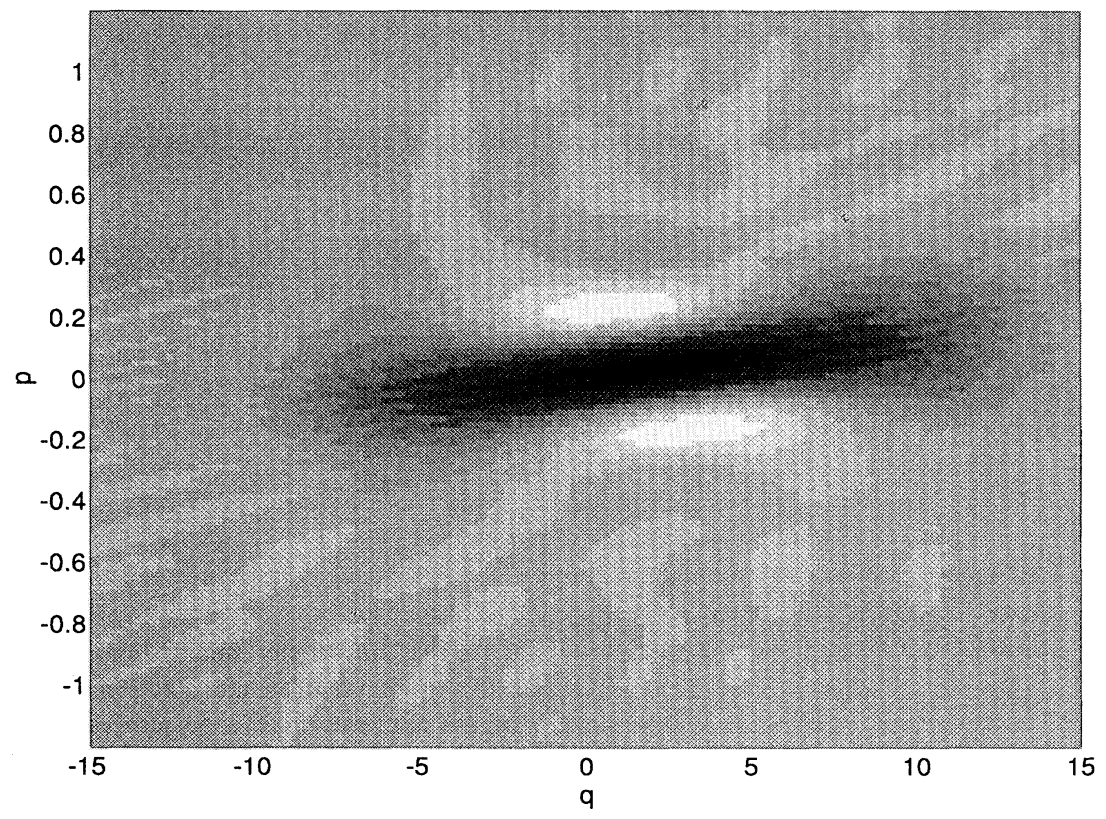

(b) 12 cycles

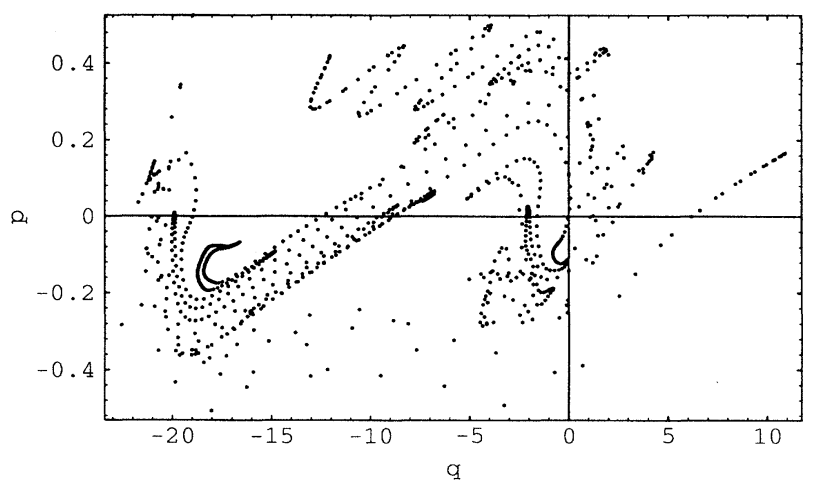

FIG. 2. (a) The contour of the Wigner function and (b) classical phase-space distribution of the hydrogenic electron at maximal strength of the application of a pulsed laser field with 12 turn-on cycles, 12 turn-off cycles, maximal electric field strength $E_{0}=10$ a.u., and angular frequency $\omega=1$ a.u. The distribution of dots in the classical distribution (b) arises from a Monte Carlo distributed set of 1000 initial conditions in space and momentum which have evolved in time via the corresponding classical Newton equation. symmetrically arranged strongly negative areas. Since the concept of negative probability is meaningless, we cannot expect to match this phase distribution with any classical model. Indeed such negative areas in the Wigner function are in general associated with quantum interference between a small number of quantum states [18].

In order to study these quantum interference effects in more detail we have considered a Floquet representation [19] of the Schrödinger equation in the $\mathrm{KH}$ frame. We start by taking the Fourier transform of the $\mathrm{KH}$ frame. Since we are working in a high-frequency, intense field regime we neglect the high-order Fourier components, and consider only the time-averaged $\mathrm{KH}$ potential

$$
V_{0}\left(\alpha_{0}, q\right)=\frac{1}{\pi} \int_{-1}^{+1} d u \frac{V\left(q+\alpha_{0} u\right)}{\sqrt{1-u^{2}}}
$$

where $\alpha_{0}=e E_{0} / m \omega^{2}$ is the maximum displacement of the electron. In this case the problem reduces to a timeindependent Schrödinger equation, which we write as

$$
\left[-\frac{\hbar^{2} \partial_{q}^{2}}{2 m}+V_{0}\left(\alpha_{0}, q\right)\right] \psi_{f}(q)=E_{f} \psi_{f}(q)
$$

The eigenvalues $E_{f}$ and eigenfunctions $\psi_{f}$ of this equation can be simply determined using the standard Numerov method [20]. The back transformation into the length gauge enables us to apply Eq. (7) to calculate the corresponding Wigner function. In Fig. 3 we have displayed the Wigner function (a) and its contour plot (b) corresponding to the symmetrical superposition of the lowest two ground states of the averaged $\mathrm{KH}$ potential $V_{0}$. We can quite clearly recognize two areas of negativity similar to those we have already observed in Fig. 2(a). Similar structures also occur for other superpositions, although the amount of negativity and the orientation may differ. Comparison of the structure in Figs. 2(a) and 3 allows us to associate the two symmetric areas of negativity observed at the peak of the pulse with the formation of superpositions of eigenstates of the time-averaged $\mathrm{KH}$ potential $V_{0}$. We note that in Fig. 3 we find none of the fringe structure that is seen in Fig. 2(a). This is because in 
(a) SUPERPOSITION OF KH EIGENSTATES

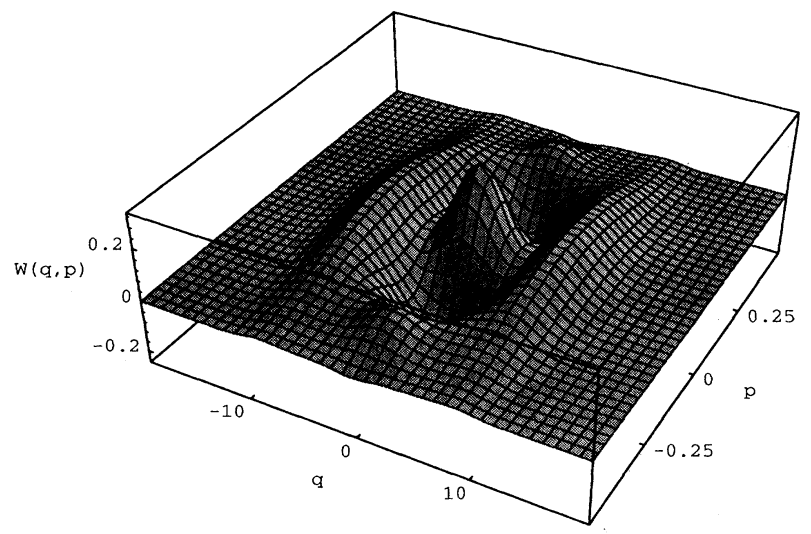

(b) SUPERPOSITION OF KH EIGENSTATES

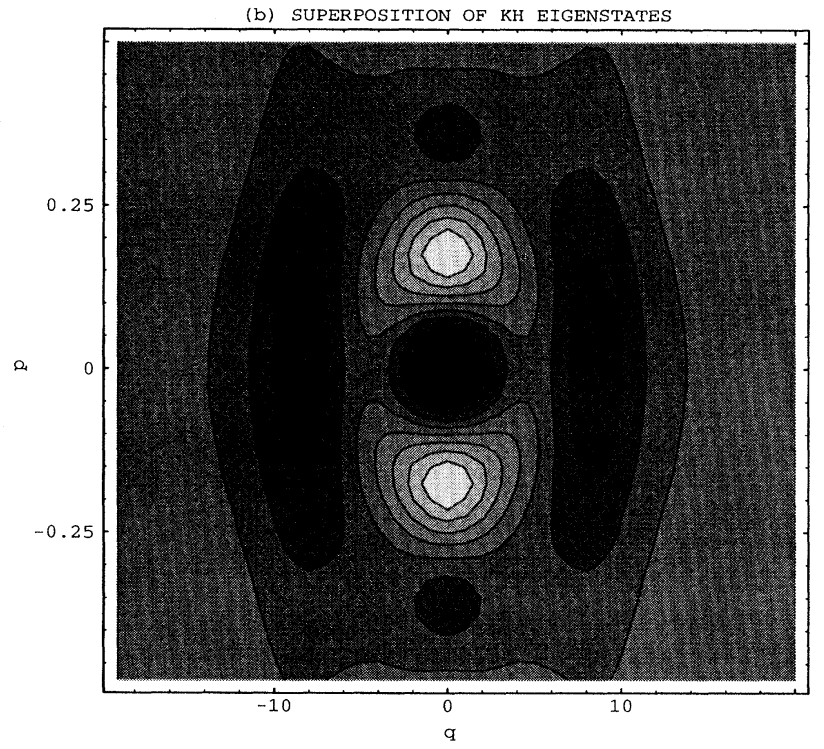

FIG. 3. The $3 d$ Wigner function (a) and its contour (b) of the symmetrical superposition of the lowest two Kramers-Henneberger eigenstates of an electron in the soft-core potential and a rapidly turned-on laser field with electric field strength $E_{0}=10$ a.u. and frequency $\omega=1$ a.u. Two symmetrically arranged areas of negativity appear due to the quantum nature of these states and in particular the coherence between the two components of the superposition.

the calculations leading to Fig. 3 we have only considered bound states of $V_{0}$ and have neglected the coupling to higher-order Fourier components of the KH potential. From this we deduce that our parameters are not such that we can neglect the higher-order components. We also note that the Floquet approach is not particularly appropriate for our timedependent pulse shape, however, this simpler analysis has allowed us to associate the negative areas in the distribution with the generation of coherent superpositions, and the fringes with ionization.

In Fig. 4 we plot the contour of the Wigner function (a) and the classical phase-space distribution after 24 laser cycles (at the end of the pulse). In this case we see the fringe structure in both the quantum and classical phase distribu-

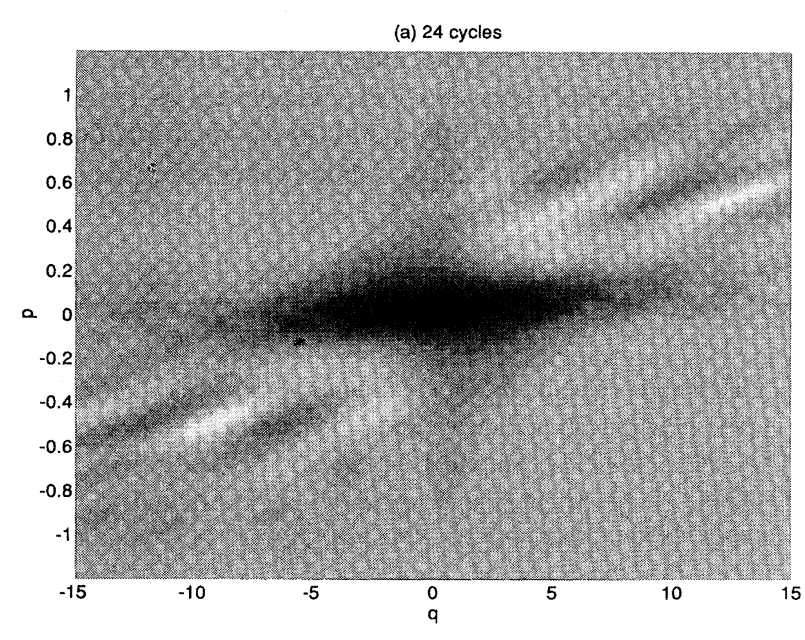

(b) 24 cycles

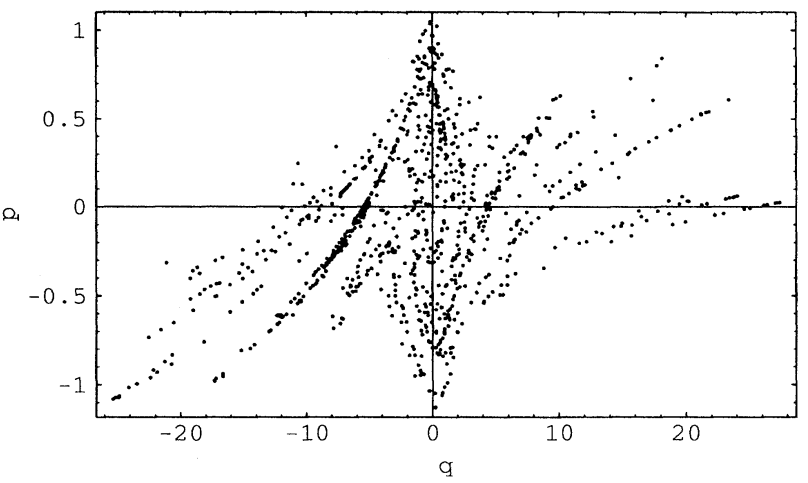

FIG. 4. (a) The contour of the Wigner function and (b) classical phase-space distribution of the hydrogenic electron immediately after the application of a pulsed laser field with 12 turn-on cycles, 12 turn-off cycles, maximal electric field strength $E_{0}=10$ a.u., and angular frequency $\omega=1$ a.u.

tions. There are negativities in the quantum case, but the length, orientation, and separation are remarkably similar in both cases. The fringes are produced in the tail of the pulse where the intensity drops below that required for stabilization. The strong agreement between the classical and quantum distributions indicates that this ionization can be described in terms of classical mechanics.

In conclusion we have observed three distinct regimes in the atomic dynamics, for a smooth pulse. During the first few cycles of the pulse there is significant ionization of the atom; this occurs until the field reaches an intensity large enough for stabilization to occur. At the peak of the pulse the high intensity and frequency allows us to consider the atom in terms of the eigenstates of the time-averaged $\mathrm{KH}$ potential. Because the field has been turned on adiabatically, only a small number of these $\mathrm{KH}$ eigenstates gain a significant population. For this reason close to the peak of the pulse, where we can make our steady-state approximation, there are strong quantum interference effects that we observe as two 
symmetric negative areas in the Wigner function. It is these quantum interference effects that lead to stabilization of the atom. Towards the end of the pulse as the intensity falls off again, the separation between $\mathrm{KH}$ eigenstates decreases, the adiabatic approach breaks down and other quantum states are populated. As the number of populated states becomes large, the quantum interference and coherence effects become small. It is for this reason that we observe such a good agreement between the quantum and classical descriptions.

The financial support of the European Community and of the U.K. Engineering and Physical Sciences Research Council is gratefully acknowledged.
[1] J. H. Eberly, Q. Su, and J. Javanainen, Phys. Rev. Lett. 62, 881 (1989); Q. Su, J. H. Eberly, and J. Javanainen, Phys. Rev. Lett. 64, 862 (1990); Q. Su and J. H. Eberly, Phys. Rev. A 43, 2474 (1991); J. H. Eberly, R. Grobe, C. K. Law, and Q. Su, in Super-Intense Laser-Atom Physics, NATO ASI Ser. B, Vol. 316, edited by B. R. M. Piraux, A. L'Huillier, and K. Rzazewski (Plenum, New York, 1993).

[2] M. V. Fedorov and A. M. Movsesian, J. Opt. Soc. Am. B 6, 928 (1989); M. V. Fedorov and M. Y. Ivanov ibid. 7, 569 (1990).

[3] V. C. Reed, P. L. Knight, and K. Burnett, Phys. Rev. Lett. 67, 1415 (1991); V. C. Reed, K. Burnett, and P. L. Knight, Phys. Rev. A 47, R34 (1993); P. L. Knight, M. Protopapas, C. Keitel, S. Vivirito, K. Burnett, V. C. Reed, and S. C. Rae, in SuperIntense Laser-Atom Physics (Ref. [1]).

[4] J. Grochmalicki, M. Lewenstein, and K. Rzazewski, Phys. Rev. Lett. 66, 1038 (1991); R. Grobe and C. K. Law, Phys. Rev. A 44, R4141 (1991); M. Gajda, J. Grochmalicki, M. Lewenstein, and K. Rzazewski, ibid. 46, 1638 (1992).

[5] M. P. de Boer, J. H. Hoogenraad, R. B. Vrijen, L. D. Noordam, and H. G. Muller, Phys. Rev. Lett. 71, 3263 (1993).

[6] M. D. Perry and J. K. Crane, Phys. Rev. A 48, R4051 (1993); A. L'Huillier and P. Balcou, Phys. Rev. Lett. 70, 774 (1993); J. J. Macklin, J. D. Kmetic, and C. L. Gordon III, ibid. 70, 766 (1993); J. Tisch, R. Smith, J. Muffett, M. Ciarrocca, J. Marangos, and M. H. R. Hutchinson, Phys. Rev. A 48, R28 (1994).

[7] A. L'Huillier, L. A. Lompre, G. Mainfray, and C. Manus, in Advances in Atomic, Molecular and Optical Physics, edited by M. Gavrila (Academic, New York, 1992); Atoms in Intense Laser Fields, edited by M. Gavrila (Academic, Boston, 1992); K. Burnett, V. C. Reed, and P. L. Knight, J. Phys. B 26, 561 (1993).

[8] See, for example, X. Tang, H. Rudolph, and P. Lambropoulos, Phys. Rev. Lett. 65, 3269 (1990); M. Pont, N. R. Walet, and M.
Gavrila, Phys. Rev. A 41, 477 (1990); M. Pont and R. Shakeshaft, ibid. 44, 4110 (1991).

[9] S. C. Rae and K. Burnett, Phys. Rev. A 48, 2490 (1993).

[10] See, for example, I. C. Percival and D. Richards, Adv. At. Mol. Phys. 11, 1 (1975); G. A. Kyrala, J. Opt. Soc. Am. B 4, 731 (1987); G. Bandarage, A. Maquet, T. Menis, R. Taieb, V. Veniard, and J. Cooper, Phys. Rev. A 46, 380 (1992); C. H. Keitel, P. L. Knight, and K. Burnett, Eur. Phys. Lett. 24, 539 (1993); C. H. Keitel and P. L. Knight, Phys. Rev. A 51, 1420 (1995).

[11] J. L. Krause, K. J. Schafer, and K. C. Kulander, Phys. Rev. Lett. 68, 3535 (1992); J. L. Krause, K. J. Schafer, and K. C. Kulander, Phys. Rev. A 45, 4998 (1992); P. B. Corkum, Phys. Rev. Lett. 71, 1994 (1993); M. Lewenstein, Ph. Balcou, M. Yu Ivanov, Anne L'Huillier, and P. B. Corkum, Phys. Rev. A 49, 2117 (1994).

[12] J. Bestle, V. A. Akulin, and W. P. Schleich, Phys. Rev. A 48, 746 (1993).

[13] E. P. Wigner, Phys. Rev. 40, 749 (1932).

[14] H. A. Kramers, Collected Scientific Papers (North-Holland, Amsterdam, 1956); W. C. Henneberger, Phys. Rev. Lett. 21, 838 (1968).

[15] T. Ménis, R. Taieb, V. Véniard, and A. Maquet, J. Phys. B 25, L263 (1992).

[16] T. Millack, J. Phys. B 26, 4777 (1993).

[17] R. Abrines and I. C. Percival, Proc. Phys. Soc. 88, 861 (1966); J. G. Leopold and I. C. Percival, J. Phys. B 12, 709 (1979).

[18] V. Bužek and P. L. Knight, in Progress in Optics, edited by E. Wolf (North-Holland, Amsterdam, in press).

[19] M. Gavrila and J. Z. Kaminski, Phys. Rev. Lett. 52, 613 (1984); M. J. Offerhaus, J. Z. Kaminski, and M. Gavrila, Phys. Lett. 112A, 151 (1985); M. Gavrila, M. J. Offerhaus, and J. Z. Kaminski, ibid. 118A, 331 (1986); J. van de Ree, J. Z. Kaminski and M. Gavrila, Phys. Rev. A 37, 4536 (1988).

[20] S. J. Koonin, Computational Physics (Benjamin/Cummings, City; CA, 1986), p. 60. 
(a) 6 cycles

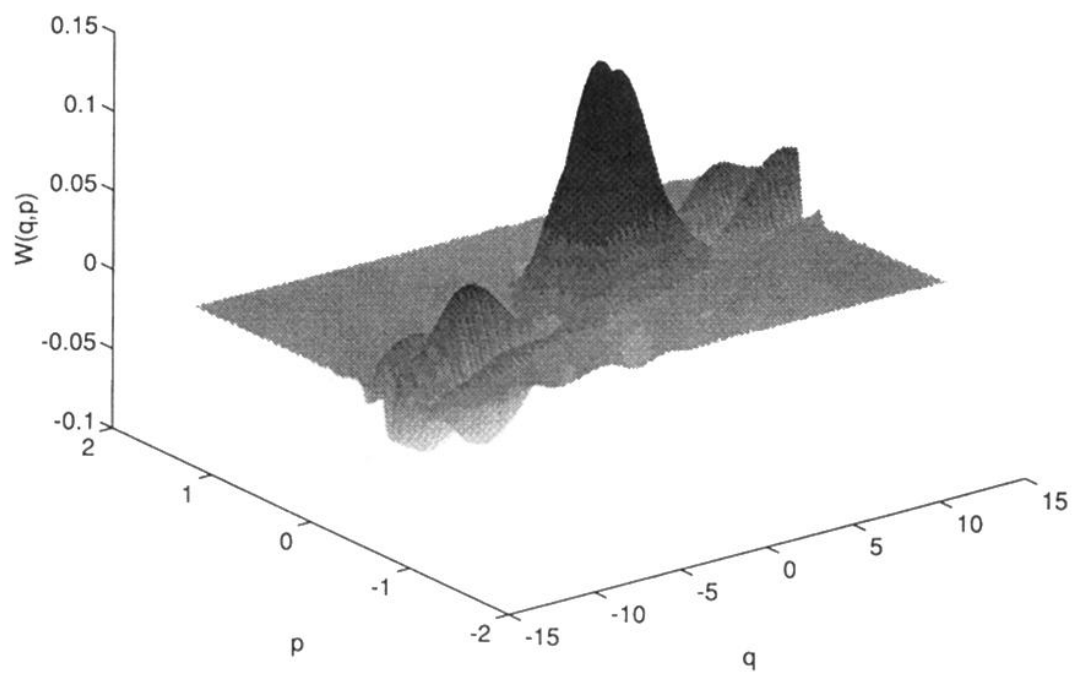

(b) 6 cycles

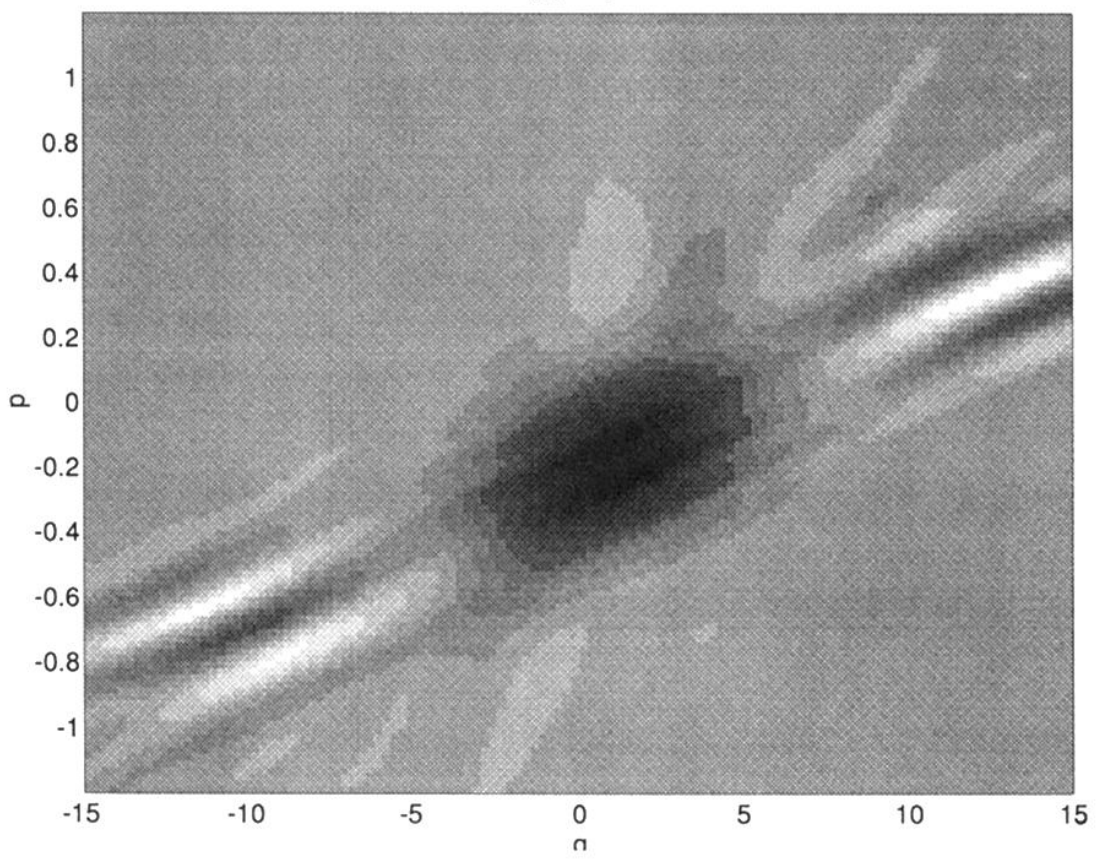

FIG. 1. The $3 d$ Wigner function (a) and its contour (b) of the hydrogenic electron after 6 cycles of the application of a pulsed laser field with 12 turn-on cycles, 12 turn-off cycles, maximal electric field strength $E_{0}=10$ a.u. and, angular frequency $\omega=1$ a.u. The quantummechanical calculations leading to these results are based on the dynamics of the hydrogenic electron in the soft-core potential as described by the corresponding Schrödinger equation. 
(a) 12 cycles

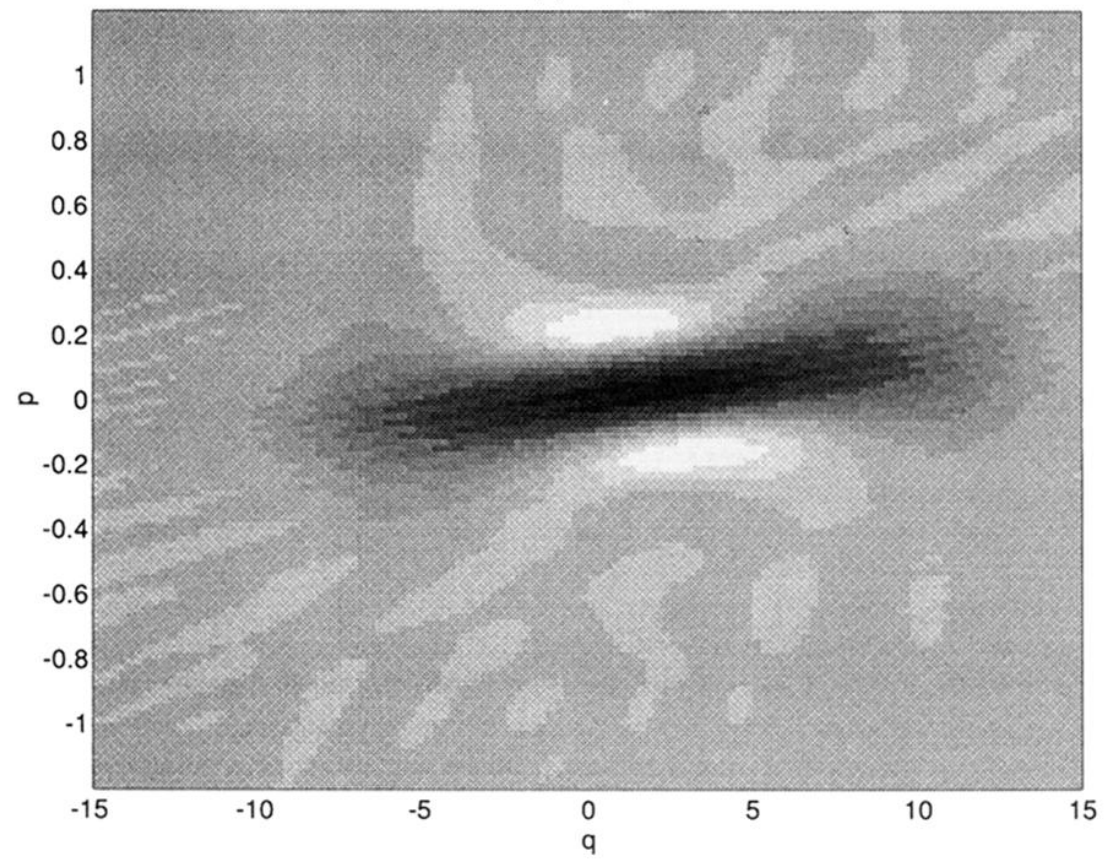

(b) 12 cycles

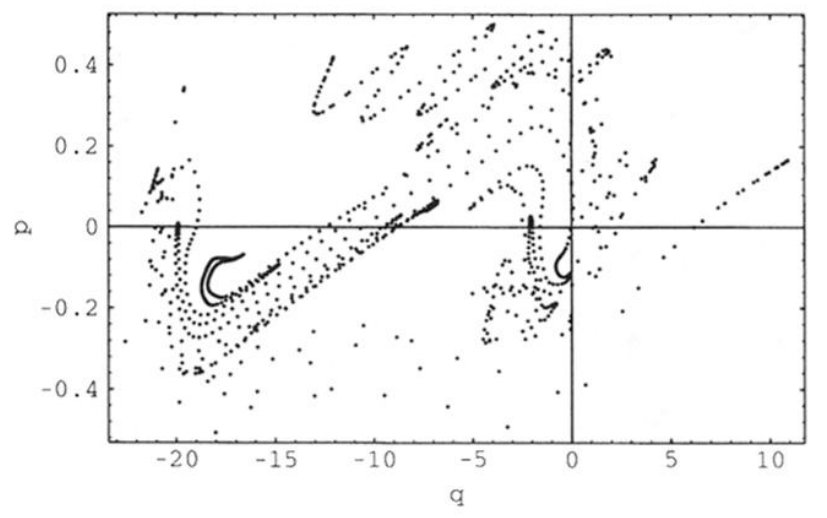

FIG. 2. (a) The contour of the Wigner function and (b) classical phase-space distribution of the hydrogenic electron at maximal strength of the application of a pulsed laser field with 12 turn-on cycles, 12 turn-off cycles, maximal electric field strength $E_{0}=10$ a.u., and angular frequency $\omega=1$ a.u. The distribution of dots in the classical distribution (b) arises from a Monte Carlo distributed set of 1000 initial conditions in space and momentum which have evolved in time via the corresponding classical Newton equation. 
(a) SUPERPOSITION OF KH EIGENSTATES
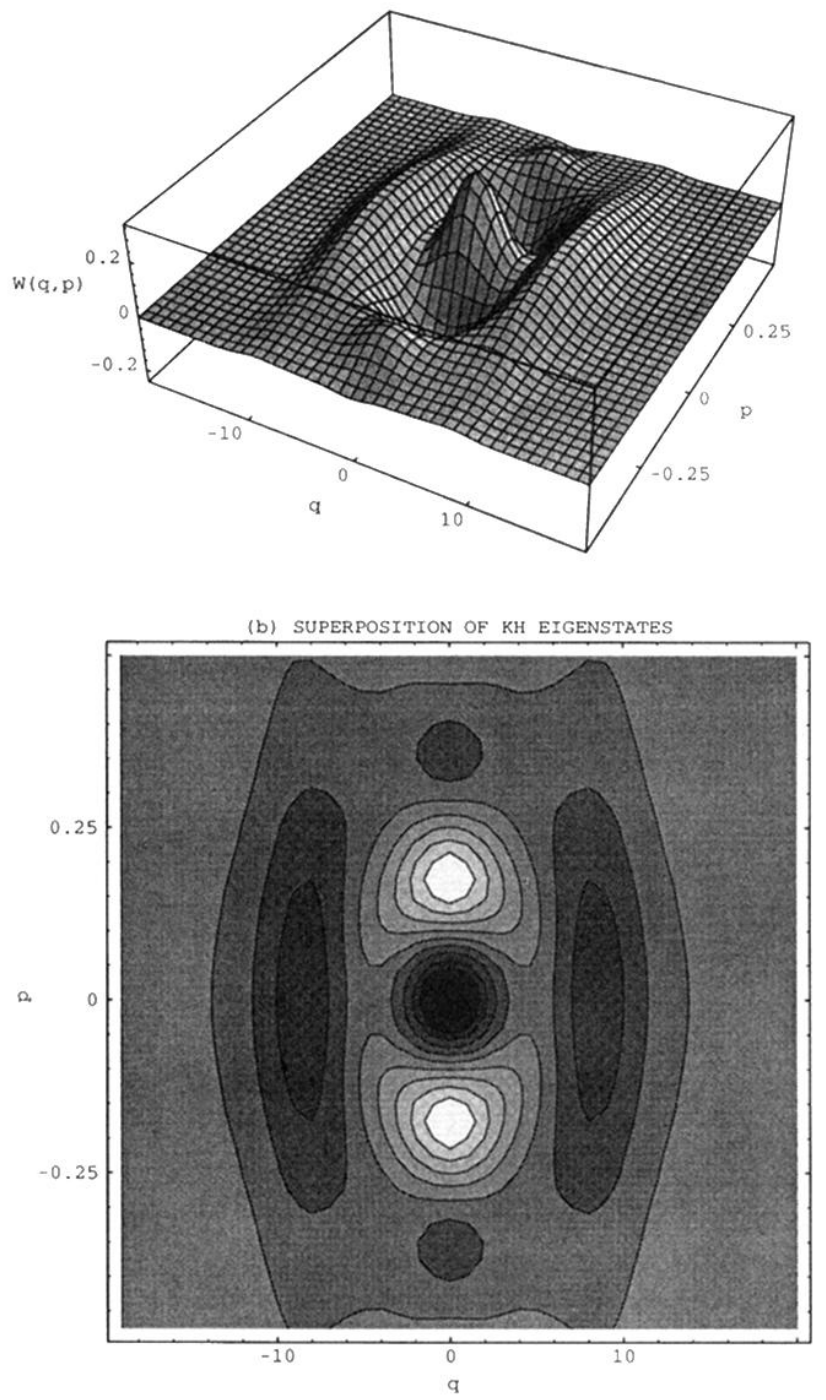

FIG. 3. The $3 d$ Wigner function (a) and its contour (b) of the symmetrical superposition of the lowest two Kramers-Henneberger eigenstates of an electron in the soft-core potential and a rapidly turned-on laser field with electric field strength $E_{0}=10$ a.u. and frequency $\omega=1$ a.u. Two symmetrically arranged areas of negativity appear due to the quantum nature of these states and in particular the coherence between the two components of the superposition. 


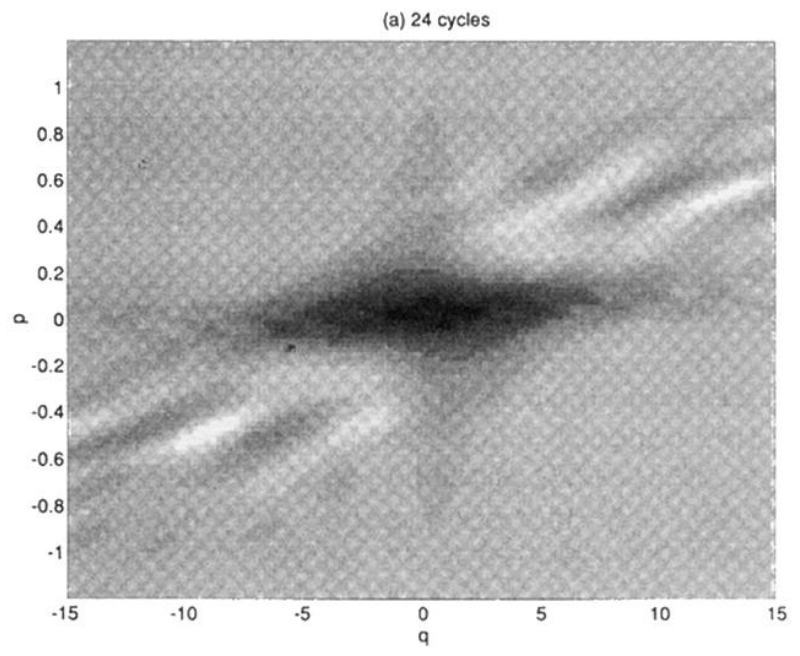

(b) 24 cycles

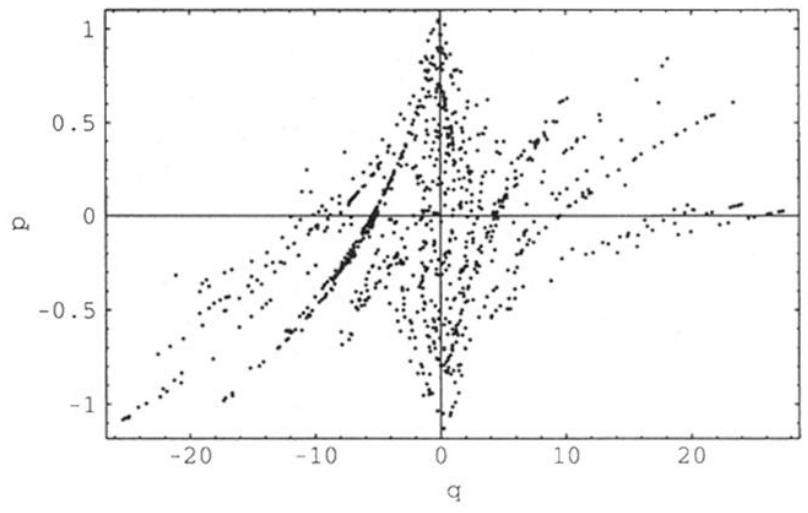

FIG. 4. (a) The contour of the Wigner function and (b) classical phase-space distribution of the hydrogenic electron immediately after the application of a pulsed laser field with 12 turn-on cycles, 12 turn-off cycles, maximal electric field strength $E_{0}=10$ a.u., and angular frequency $\omega=1$ a.u. 